\title{
Improvements in practice from assessing standards of recording psychiatric case-notes
}

\author{
Tom O'Hare
}

The case-notes of all patients in a district general adult psychiatric unit were assessed for standards of recording selected key liems. The results were presented at a departmental audif meeting, and the assessment repeated after four months. Initial audit revealed poor standards of notekeeping in certain areas. On repeat assessment, standards of documentation had improved for all the key liems assessed, and reached statistical significance for physical examination. This audit was quick, easy and cheap, revealed unexpectedly poor standards of notekeeping, and evidently produced a measurable and significant improvement in practice.

Well kept medical case-notes are crucial in ensuring high quality care, as well as being an important source of medico-legal information.

The purpose of this audit was to examine the standard of recording certain key items of information in in-patients' case-notes. Absence of these items implies that procedures may not have been carried out, or that important aspects of patient management have not been considered. Hence, in addition to measuring directly the standard of record keeping, this audit gave an indirect measure of standards of patient care.

\section{The study}

On 30 March 1994, a survey was made of the medical case-notes of all in-patients in the three wards of a district general hospital adult psychiatric unit. Records for the current admission were scrutinised with reference to documentation of mental state, physical examination, diagnosis, and management plan.

The procedure was repeated four months later, on 18 July 1994 , to assess the impact of the audit. Patients, staff and wards were not identified.

\section{Findings}

Results of initial assessment (Table 1)

Thirty-eight sets of case-notes were examined.

Mental state. All patients had documentation of mental state on admission. Three of them had been in-patients for a number of years, and none of these had had a mental state examination recorded within the past year.

Physical examination. For 13 of the 38 patients (34\%), no record of any attempt at physical examination could be found in their notes. Three

Table 1. Results of serial assessment of case-notes

\begin{tabular}{|c|c|c|c|}
\hline & $\begin{array}{l}30.3 .94 \\
n=38\end{array}$ & $\begin{array}{l}18.7 .94 \\
n=39\end{array}$ & $\begin{array}{l}\chi^{2} \\
\text { (Yates-corrected) }\end{array}$ \\
\hline $\begin{array}{l}\text { Mental state: none documented in past year } \\
\text { Physical examination: }\end{array}$ & $3(8 \%)$ & $2(5 \%)$ & NS \\
\hline Complete documentation & $15(39 \%)$ & $26(67 \%)$ & $P<0.05$ \\
\hline No physical and no reason given & $13(34 \%)$ & $2(5 \%)$ & $P<0.01$ \\
\hline Incompletely documented & $9(24 \%)$ & $6(15 \%)$ & NS \\
\hline Stated 'patient refused' & 1 (3\%) & $4(10 \%)$ & NS \\
\hline Diagnosis: none in current admission/volume & 3 (8\%) & $2(5 \%)$ & NS \\
\hline Management plan: none in current admission/volume & $6(16 \%)$ & 1 (3\%) & NS \\
\hline
\end{tabular}


(8\%) of these were long-term in-patients, for whom no examination had been recorded within the past year (older volumes of their notes were not scrutinised). One patient was on record as having refused examination, although for the remainder no reason was given for failing to record physical examination.

Of the 22 who did have some record of physical examination, nine were judged incomplete: three had only 'pulse, blood pressure and heart sounds'; four had no record of any examination of their nervous system, and a further two had evidently received only a very brief nervous system examination, with significant omissions. In only one of these was a reason given for incomplete examination ('patient uncooperative'). One further patient was recorded as having refused physical examination. Only $15(39 \%)$ patients were judged to have documentation of a full physical examination.

Diagnosis. Three $(8 \%)$ patients had no diagnosis written in their notes for the current admission. Two of these were long-stay patients, for whom no diagnosis could be found in their current volume of notes (covering at least one year prior to the survey).

Management plan. Six (16\%) patients did not have a management plan written in their notes for the current admission. Three of these were the long-stay patients, for whom this again referred to the current volume.

\section{Comment}

In general, the standards of recording mental state and diagnosis were good. However, several deficiencies were revealed.

Physical examination. An unacceptably high proportion (34\%) of patients had no record of any physical examination, and a further $24 \%$ had only partial documentation. In only two cases was the incompleteness qualified by an explanatory note. Examination of the nervous system was particularly likely to be omitted or curtailed.

There are various possible reasons why the recording of physical examination was inadequate. The admitting doctor may have omitted or curtailed the examination. This could have been due to lack of time, fatigue, or a perception that the examination was unimportant. The examination may have been performed but not recorded. The patient may have been uncooperative, but the doctor failed to record this, either through forgetfulness or through lack of appreciation of its importance.

Long-stay patients. This group of patients (of whom there were only three) tended not to have any record in their current volume of notes (covering over a year prior to survey) of diagnosis, management plan. physical or mental state examination. For these patients, the hospital ward is their home. They are entitled to recetve such general medical care as they would be offered were they living outside hospital, which would include opportunistic health screening. Although the audit did not address this issue, it was agreed that this was a possible shortfall in the service.

Management plan. Three (10\%) of the acute patients had no documentation of even a simple management plan, which suggests that this aspect of notekeeping may sometimes not be seen as important by the admitting doctor, or may alternatively reflect uncertainty due to a lack of expertise in the specialty.

\section{Action taken}

The above findings were discussed at a departmental medical audit meeting on 30 March 1994, with all trainees except one present. It was agreed that the frequency of recording physical examination was unacceptably low, and that special attention should be paid to this in future. The medicolegal importance of documenting when a patient had refused a physical examination was emphasised.

Although the other measures of performance were generally good, it was agreed that patients should always have a written management plan, and that long-stay patients' case-notes tended to be uninformative. In order to measure whether the audit had led to a change in practice, I announced that I would repeat the survey in about four months.

\section{Results of follow-up audit ('closing the loop') (Table 1)}

Thirty-nine sets of notes were studied. Three of these patients were present at the first audit. There were no changes of staff between the two assessments.

Mental state. Two patients had no documentation of mental state in their current notes folder: 
both were long-stay patients present at the previous audit.

Physical examination. Twenty-six (67\%) had good documentation, and four (10\%) had no physical examination but were documented as having refused. A further six (15\%) were well documented apart from a very brief comment on central nervous system. One had only 'pulse, blood pressure and heart sounds', but was present at the previous audit. Two $(5 \%)$ had no physical examination recorded in their current volume: both were long-stay patients.

Diagnosis. Two (5\%) had no diagnosis: neither was a long-stay patient.

Management plan. One had no documentation and was a long-stay patient.

\section{Conclusion}

The repeat audit showed an improvement in all measures. In particular, the documentation of physical examination was markedly better, with statistically significant changes. However, there were still $15 \%$ of patients with only partial documentation, usually an incomplete central nervous system examination.

It appears likely that this measurable improvement in practice occurred as a result of the audit process. Other possible reasons include chance variation, or increasing experience of senior house officers in their first psychiatry posts (although the improvements were not confined to them). It was recognised that the regular six-monthly change-over of trainees could be a future source of deterioration in standards. To attempt to preserve the improvement, guidelines on recording case-notes were subsequently included in the new junior doctors' handbook.

Poor notekeeping is a fairly widespread problem in many units. This audit was quick, easy and cheap, and evidently produced a measurable and significant improvement in practice.

Tom O'Hare, Senior Registrar in Psychiatry. Edenfield Centre, Mental Health Services, Bury New Road, Prestwich, Manchester M25 3BL 